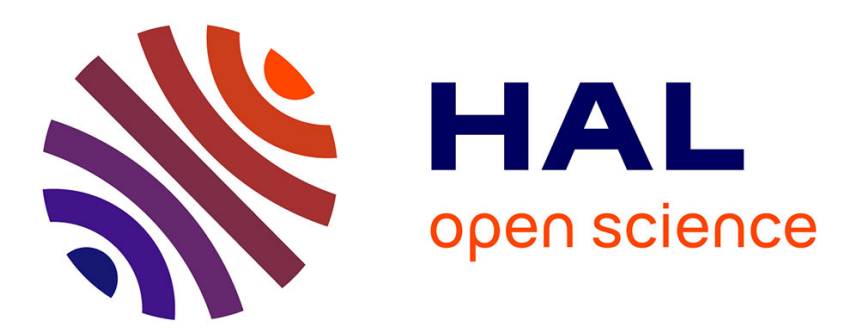

\title{
Strict Lyapunov-Krasovskiı̌ Functionals for undirected networks of Euler-Lagrange systems with time-varying delays
}

\author{
Emmanuel Nuno, Ioannis Sarras, Antonio Loria, Mohamed Maghenem, \\ Emmanuel Cruz-Zavala, Elena Panteley
}

\section{To cite this version:}

Emmanuel Nuno, Ioannis Sarras, Antonio Loria, Mohamed Maghenem, Emmanuel Cruz-Zavala, et al.. Strict Lyapunov-Krasovskiı̌ Functionals for undirected networks of Euler-Lagrange systems with timevarying delays. Systems and Control Letters, 2020, 135, pp.104579. 10.1016/j.sysconle.2019.104579 . hal-02414136

HAL Id: hal-02414136

https://hal-centralesupelec.archives-ouvertes.fr/hal-02414136

Submitted on 17 Jul 2020

HAL is a multi-disciplinary open access archive for the deposit and dissemination of scientific research documents, whether they are published or not. The documents may come from teaching and research institutions in France or abroad, or from public or private research centers.
L'archive ouverte pluridisciplinaire HAL, est destinée au dépôt et à la diffusion de documents scientifiques de niveau recherche, publiés ou non, émanant des établissements d'enseignement et de recherche français ou étrangers, des laboratoires publics ou privés. 


\title{
Strict Lyapunov-Krasovskil Functionals for Undirected Networks of Euler-Lagrange Systems with Time-varying Delays
}

\author{
Emmanuel Nuño ${ }^{\mathrm{a}}$, Ioannis Sarras ${ }^{\mathrm{b}}$, Antonio Loría $^{\mathrm{c}}$, Mohamed Maghenem $^{\mathrm{d}}$, Emmanuel Cruz-Zavala $^{\mathrm{a}}$, Elena Panteley ${ }^{\mathrm{d}, \mathrm{c}}$ \\ ${ }^{a}$ Department of Computer Science, CUCEI, University of Guadalajara. Guadalajara, Mexico \\ ${ }^{b}$ DTIS, ONERA, Université Paris-Saclay, F-91123 Palaiseau, France \\ ${ }^{c}$ Laboratoire des Signaux et Systèmes, CNRS, Gif-sur-Yvette, France. \\ ${ }^{d}$ Department of Computer Engineering, University of California Santa Cruz. California, USA. \\ ${ }^{e} I T M O$ University, Kronverkskiy av. 49, Saint Petersburg, 197101, Russia.
}

\begin{abstract}
For an undirected network of nonidentical interconnected Euler-Lagrange systems whose communication is affected by varying time-delays that may not be differentiable, we consider the problem of establishing leaderless and leader-follower consensus via the simplest Proportional plus damping decentralized controller. The main contribution of this work is to prove that the agents' positions and velocities converge uniformly, globally, and asymptotically to a common non-specified position in the leaderless case, and to a given reference in the leader-follower case. The main results are established via Lyapunov's direct method; a Strict Lyapunov-Krasovskiü Functional is constructed, to the best of our knowledge, for the first time in the literature. It is shown that the resulting closed-loop system is Input-to-State Stable with regards to external additive inputs (perturbations). In turn, the separation principle applies to a certainty-equivalence controller, implemented with any globally convergent velocity estimator, such as the Immersion \& Invariance observer.
\end{abstract}

Keywords: Euler-Lagrange Dynamics; Multi-Agent Systems; Time-Delays; Strict Lyapunov Functions.

\section{Introduction}

It is said that Euler-Lagrange (EL) systems achieve consensus if all their generalized positions converge to a common value. We identify two particular consensus problems: leader-follower, in which case an agreement trajectory is given as a common reference [19] and the leaderless, in which case the agents' positions converge to a common non-specified value $[6,7]$. The practical applications of consensus of EL-agents are diverse and can be found in different fields of mechanical, electrical and electromechanical systems such as, for example, in robotics [8] or in control of aerial vehicles [28]. Consensus problems may be and have been solved via decentralized feedback of the agents' neighbors' positions and, possibly, their velocities. Pioneer works addressing the consensus problem for EL systems include [1-5]. Notably, the simplest consensus controller is the Proportional plus damping $(\mathrm{P}+\mathrm{d})$ scheme reported in [5]; moreover, it is showed in [18] that this controller works also with directed interconnection topologies.

Consensus control for networked-interconnected systems is, however, typically stymied by the effect of interconnection delays. This problem has been addressed, for instance, in $[9-15]$. See also $[16,17]$ where the results of $[5]$ are extended by considering the presence of interconnection

\footnotetext{
Nuño)

Email address: emmanuel.nuno@cucei.udg.mx (Emmanuel
}

delays. A common drawback of all these works, nonetheless, is that they rely on the assumption that the timevarying delays are differentiable and that their derivative is bounded. Clearly, this imposes a practical limitation because several communication media (such as Internet) do not ensure this hypothesis. Remarkably, in $[19,20]$ the leader-follower (in the former) and the leaderless (in the latter) synchronization problems are solved under the assumption that delays may be non-differentiable. Moreover, in both references it is assumed that the systems' parameters are unknown hence, the convergence of the consensus errors is ensured via adaptive control.

The main contribution of this paper is the design of an original strict Lyapunov-Krasovskiü functional to guarantee uniform global asymptotic consensus for a network of EL-systems controlled by decentralized $\mathrm{P}+\mathrm{d}$ controllers in the presence of, possibly non-differentiable, time-varying delays and under an undirected-graph interconnection topology. With this proposal we are able to reveal new properties of the simplest available control laws $(\mathrm{P}+\mathrm{d})$ for consensus of EL-systems, despite the fact that these have been exhaustively studied and implemented in the literature (see $[5,16-18]$ ).

The importance of having a strict Lyapunov-Krasovskii functional cannot be overestimated; it allows to prove uniform global asymptotic consensus. Furthermore, we establish input-to-state stability for the closed-loop system. This is in clear contrast with previous works in which only 
convergence is established. Indeed, the latter is a weaker property that does not guarantee robustness with respect to bounded disturbances.

We also stress that establishing input-to-state stability is well beyond pure academic interest. As we show, in addition, a byproduct of our main statements is an extension to the case in which velocity measurements are replaced by estimates obtained from a converging velocity observer that guarantees uniform exponential convergence of the observation errors, such as the Immersion \& Invariance (I\&I) velocity observer [22], to render the delaydependent stability condition decentralized - cf. [16, 17], and to prove Input-to-State Stability (ISS) with respect to external additive inputs (perturbations). See also [29] where a partial-state feedback controller under constant delays is proposed.

Although we are not aware of other articles in which the consensus problem is treated under the exact same setting and where input-to-state stability is established, a clear drawback of our main results, that contrasts with [19] and [20], is that we assume that part of the robots' parameters are known and, more importantly, that the interconnection graph is undirected.

The rest of the paper is organized as follows. In the following section we present some background material on the systems' models and lay our main hypotheses. In Sections 3 and 4 we present our main results: on leaderless, leaderfollower, and output-feedback consensus respectively. We conclude with some remarks in Section 5.

\section{Background}

Throughout the paper, the following notation is employed. $\mathbb{R}:=(-\infty, \infty), \mathbb{R}_{>0}:=(0, \infty), \mathbb{R}_{\geq 0}:=[0, \infty)$ and $\mathbb{N}:=\{1,2,3, \ldots\} . \mathbf{I}_{n} \in \mathbb{R}^{n \times n}$ denotes the $n \times n$ identity matrix, $\mathbf{1}_{n} \in \mathbb{R}^{n}$ defines the vector of $n$ elements equal to one and $\mathbf{0}_{n} \in \mathbb{R}^{n}$ is the all-zeros vector. For any $\mathbf{x} \in \mathbb{R}^{n}$, $\nabla_{\mathbf{x}}:=\left[\partial_{x_{1}}, \ldots, \partial_{x_{n}}\right]^{\top}$ stands for the gradient operator of a scalar function and $|\mathbf{x}|$ for the Euclidean norm. $\lambda_{m}\{\mathbf{A}\}$ and $\lambda_{M}\{\mathbf{A}\}$ are the minimum and the maximum eigenvalues of the symmetric matrix $\mathbf{A} \in \mathbb{R}^{n \times n} . \bar{N}:=\{1,2, \ldots, N\}$ for $N \in \mathbb{N}$. A continuous function $\gamma: \mathbb{R}_{\geq 0} \mapsto \mathbb{R}_{\geq 0}$ is of class $\mathcal{K}$ if $\gamma(0)=0$ and it is strictly increasing. Further, it is of class $\mathcal{K}_{\infty}$ if it is of class $\mathcal{K}$ and unbounded. For an absolutely continuous function $\phi:[-h, 0] \rightarrow \mathbb{R}^{n}, h>0$, we define

$$
\|\phi\|:=\max _{-h \leq \theta \leq 0}|\phi(\theta)|
$$

and we denote by $\mathcal{C}[-h, 0]$ the space of such functions.

The norm defined in (1) is used in this paper to formulate conditions for uniform global asymptotic stability of the null solution of functional differential equations

$$
\dot{x}=f\left(t, x_{t}\right), \quad f(t, 0) \equiv 0,
$$

where $x_{t}$ is short-hand notation for $x_{t}(\theta):=x(t+\theta)$ for all $\theta \in[-h, 0], f: \mathbb{R}_{\geq 0} \times \mathbb{R}^{n} \times \mathcal{C}[-h, 0] \rightarrow \mathbb{R}^{n}$ is continuous and locally Lipschitz in the second argument.
Remark 1. We observe that in the first works on stability of solutions of systems (2) by N. N. Krasovskiu, some of which are compiled in [27], the $\mathcal{L}_{2}$ norm of $\phi(\theta)$ is used instead of the $\max (\cdot)$.As in [26], we use (1), which results convenient to formulate the following statement.

Theorem 1. - cf. $[26 \text {, Theorem 1.3 }]^{1}$ Let $\beta_{i}: \mathbb{R}_{\geq 0} \rightarrow$ $\mathbb{R}_{\geq 0}$ be class $\mathcal{K}_{\infty}$ functions. If there exists a continuous differentiable functional $\mathcal{V}: \mathbb{R}_{\geq 0} \times \times \mathcal{C}[-h, 0] \rightarrow \mathbb{R}_{\geq 0}$ such that

$$
\beta_{1}\left(\left\|x_{t}(0)\right\|\right) \leq \mathcal{V}\left(t, x_{t}\right) \leq \beta_{2}\left(\left\|x_{t}\right\|\right),
$$

and

$$
\dot{\mathcal{V}}\left(t, x_{t}\right) \leq-\beta_{3}\left(\left\|x_{t}(0)\right\|\right)
$$

then the trivial solution of (1) is uniformly globally asymptotically stable.

Remark 2. Functionals $\mathcal{V}$ satisfying the above are commonly referred to as Strict Lyapunov Krasovskiu functionals.

\subsection{Euler-Lagrange Agents}

We consider a network composed of $N$ fully-actuated and conservative EL-agents, with $n$-Degrees-of-Freedom (DoF). The dynamics of the $i$ th-agent is given by

$$
\frac{d}{d t} \nabla_{\dot{\mathbf{q}}_{i}} \mathcal{L}_{i}\left(\mathbf{q}_{i}, \dot{\mathbf{q}}_{i}\right)-\nabla_{\mathbf{q}_{i}} \mathcal{L}_{i}\left(\mathbf{q}_{i}, \dot{\mathbf{q}}_{i}\right)=\tau_{i}, \quad i \in \bar{N}
$$

where $\mathcal{L}_{i}\left(\mathbf{q}_{i}, \dot{\mathbf{q}}_{i}\right)$ is the Lagrangian function that is defined as $\mathcal{L}_{i}\left(\mathbf{q}_{i}, \dot{\mathbf{q}}_{i}\right):=\mathcal{K}_{i}\left(\mathbf{q}_{i}, \dot{\mathbf{q}}_{i}\right)-\mathcal{U}_{i}\left(\mathbf{q}_{i}\right)$, with $\mathcal{K}_{i}\left(\mathbf{q}_{i}, \dot{\mathbf{q}}_{i}\right):=$ $\frac{1}{2} \dot{\mathbf{q}}_{i}^{\top} \mathbf{M}_{i}\left(\mathbf{q}_{i}\right) \dot{\mathbf{q}}_{i}$ the kinetic energy and $\mathcal{U}_{i}\left(\mathbf{q}_{i}\right)$ the potential energy. $\mathbf{q}_{i}, \dot{\mathbf{q}}_{i} \in \mathbb{R}^{n}$ are the generalized position and velocity, respectively, $\mathbf{M}_{i}\left(\mathbf{q}_{i}\right) \in \mathbb{R}^{n \times n}$ is the generalized inertia matrix, which is symmetric positive definite, and $\tau_{i} \in \mathbb{R}^{n}$ is the control input.

The EL-equations of motion (5) can be written in a compact form as

$$
\mathbf{M}_{i}\left(\mathbf{q}_{i}\right) \ddot{\mathbf{q}}_{i}+\mathbf{C}_{i}\left(\mathbf{q}_{i}, \dot{\mathbf{q}}_{i}\right) \dot{\mathbf{q}}_{i}+\nabla_{\mathbf{q}_{i}} \mathcal{U}_{i}\left(\mathbf{q}_{i}\right)=\tau_{i},
$$

where $\mathbf{C}_{i}\left(\mathbf{q}_{i}, \dot{\mathbf{q}}_{i}\right) \in \mathbb{R}^{n \times n}$ is the Coriolis and centrifugal forces matrix, defined via the Christoffel symbols of the first kind. We restrict to EL-agents (6) that satisfy the following assumption:

Assumption A1: There exist $m_{1 i} \in \mathbb{R}_{>0}$ and $m_{2 i} \in \mathbb{R}_{>0}$ such that, for all $\mathbf{q}_{i} \in \mathbb{R}^{n}, m_{1 i} \mathbf{I}_{n} \leq \mathbf{M}_{i}\left(\mathbf{q}_{i}\right) \leq m_{2 i} \mathbf{I}_{n}$.

This assumption is ubiquitous in robot control but it also holds for other different physical systems, see [23, 24]. Further, model (6) has the following properties [21]:

Property P1: Matrix $\dot{\mathbf{M}}_{i}\left(\mathbf{q}_{i}\right)-2 \mathbf{C}_{i}\left(\mathbf{q}_{i}, \dot{\mathbf{q}}_{i}\right)$ is skewsymmetric. Further, $\dot{\mathbf{M}}_{i}\left(\mathbf{q}_{i}\right)=\mathbf{C}_{i}\left(\mathbf{q}_{i}, \dot{\mathbf{q}}_{i}\right)+\mathbf{C}_{i}^{\top}\left(\mathbf{q}_{i}, \dot{\mathbf{q}}_{i}\right)$. $\triangleleft$

Property P2: There exists $k_{c i} \in \mathbb{R}_{>0}$ such that, for all $\mathbf{q}_{i} \in \mathbb{R}^{n},\left\|\mathbf{C}_{i}\left(\mathbf{q}_{i}, \dot{\mathbf{q}}_{i}\right) \dot{\mathbf{q}}_{i}\right\| \leq k_{c i}\left\|\dot{\mathbf{q}}_{i}\right\|^{2}$.

\footnotetext{
${ }^{1}$ In its original form, in [27], this theorem is formulated for asymptotic stability.
} 


\subsection{Interconnection Topology}

As it is customary, we use graphs to represent the interconnection topology among the $N$ EL-agents. In particular, we employ the graph Laplacian matrix $\mathbf{L}:=\left\{L_{i j}\right\} \in$ $\mathbb{R}^{N \times N}$ that is defined as $L_{i i}=\sum_{j \in \mathcal{N}_{i}} a_{i j}$ and $L_{i j}=-a_{i j}$, where $a_{i j}>0$ if $j \in \mathcal{N}_{i}$ and $a_{i j}=0$ otherwise. The set $\mathcal{N}_{i}$ contains all the neighbors of the $i$ th-EL-agent. Note that, by construction, $\mathbf{L}$ has a zero row sum. Therefore $\mathbf{L} \mathbf{1}_{N}=\mathbf{0}_{n}$.

We assume that the agents exchange information according to the following assumption.

Assumption A2: The EL-agents interconnection graph is undirected, static and connected.

From A2, the Laplacian $\mathbf{L}$ is symmetric; positive semidefinite; it has a single zero-eigenvalue, with the associated eigenvector $\mathbf{1}_{N}$, and all of the other eigenvalues are strictly positive; and $\operatorname{rank}(\mathbf{L})=N-1$. Further, $\operatorname{ker}(\mathbf{L})=\alpha \mathbf{1}_{N}$, $\forall \alpha \in \mathbb{R}$.

We also consider the fact that the information exchange between agents is subjected to time-varying delays; for these interconnection delays we assume the following.

Assumption A3: The communications, from the $j$-th agent to the $i$-th agent, is subjected to a variable timedelay $T_{j i}(t)$ with a known upper-bound ${ }^{*} T_{j i}$, that is,

$$
0 \leq T_{j i}(t) \leq{ }^{*} T_{j i}<\infty
$$

Remark 3. One of the main contributions of this work is that the time-varying delay $T_{j i}(t)$ is not required to be differentiable.

In addition to $\mathbf{A 2}$, we make the following assumption for the leader-follower interconnection.

Assumption A4: There is a nonempty set of followers that have a direct access to the desired constant position $\mathbf{q}_{\ell}$.

Next, let us define a diagonal matrix $\mathbf{A}_{\ell}:=\operatorname{diag}\left\{a_{i \ell}\right\} \in$ $\mathbb{R}^{N \times N}$ to model the leader-follower interconnections, such that $a_{i \ell}>0$ if node $i$ receives the leader desired position and $a_{i \ell}=0$, otherwise. Define $\mathcal{N}_{\ell}$ as the set of all follower agents that receive the leader desired position. The following lemma, which is a special case of Lemma 3 in [25] and Lemma 1.6 of [7], provides a fundamental property of the composed Laplacian matrix $\mathbf{L}_{\ell}:=\mathbf{L}+\mathbf{A}_{\ell}$.

Lemma 1. Consider the matrix $\mathbf{A}_{\ell}:=\operatorname{diag}\left\{a_{i \ell}\right\} \geq 0 \in$ $\mathbb{R}^{N \times N}$ and suppose that $\mathcal{N}_{\ell}$ is non-empty. Assume that $\mathbf{A} \mathbf{2}$ holds, then the matrix $\mathbf{L}_{\ell}$ is symmetric, positive definite and of full rank.

Note that A4 ensures that there is at least one agent receiving information from its neighbors.

\section{Leaderless Consensus}

We speak of leaderless consensus if there exists $\mathbf{q}_{c} \in \mathbb{R}^{n}$ such that

$$
\lim _{t \rightarrow \infty}\left|\dot{\mathbf{q}}_{i}(t)\right|=0, \quad \lim _{t \rightarrow \infty} \mathbf{q}_{i}(t)=\mathbf{q}_{c}
$$

for all $i \in[0, N]$. In [17] it has been shown that the $\mathrm{P}+\mathrm{d}$ controller

$$
\tau_{i}=\nabla_{\mathbf{q}_{i}} \mathcal{U}_{i}\left(\mathbf{q}_{i}\right)-d_{i} \dot{\mathbf{q}}_{i}-p_{i} \sum_{j \in \mathcal{N}_{i}} a_{i j}\left(\mathbf{q}_{i}-\mathbf{q}_{j}\left(t-T_{j i}(t)\right)\right),
$$

where $p_{i}, d_{i} \in \mathbb{R}_{>0}$ are the proportional and the damping gains, respectively, guarantees leaderless consensus provided that the control gains satisfy

$$
2 d_{i}>p_{i} \sum_{j \in \mathcal{N}_{i}} a_{i j}\left(\alpha_{i}+\frac{{ }^{*} T_{j i}^{2}}{\alpha_{j}}\right)
$$

for some $\boldsymbol{\alpha}:=\left[\alpha_{1}, \ldots, \alpha_{N}\right]^{\top} \in \mathbb{R}^{N}$ such that $\alpha_{i}>0$.

Nonetheless, the main statement in [17] has two significant limitations. Firstly, that the mere convergence properties (8) do not guarantee robustness with respect to bounded disturbances, a property that, in contrast, is guaranteed by uniform asymptotic stability [27]. Secondly, and more importantly, it is assumed in the latter reference that the time-delays are differentiable. As it appears, both limitations may be lifted, by using Lyapunov's direct method. Indeed, our main contribution is to provide a strict Lyapunov-Krasovskiu functional to establish the following statement.

Proposition 1. For each $i \in \bar{N}$, consider the system (6) in closed loop with (9) and let (10) hold. Then, under Assumptions $\mathbf{A 1}-\mathbf{A 3}$, there exists $\mathbf{q}_{c} \in \mathbb{R}^{n}$ such that the equilibrium of the closed-loop system $(\dot{\mathbf{q}}, \mathbf{q})=\left(\mathbf{0}, \mathbf{1}_{N} \otimes \mathbf{q}_{c}\right)$, where $\mathbf{q}:=\left[\mathbf{q}_{1}^{\top} \cdots \mathbf{q}_{N}^{\top}\right]^{\top}$, is uniformly globally asymptotically stable. Furthermore, the closed-loop system is Input-to-State stable with respect to additive inputs $\mathbf{u}:=$ $\left[\mathbf{u}_{1}^{\top} \cdots \mathbf{u}_{N}^{\top}\right]^{\top}$.

Proof. We start by deriving the closed-loop equations; after (6) and (9) we have

$$
\begin{aligned}
\ddot{\mathbf{q}}_{i}= & -\mathbf{M}_{i}^{-1}\left(\mathbf{q}_{i}\right)\left[\mathbf{C}_{i}\left(\mathbf{q}_{i}, \dot{\mathbf{q}}_{i}\right) \dot{\mathbf{q}}_{i}+d_{i} \dot{\mathbf{q}}_{i}+p_{i} \mathbf{e}_{i}\right] \\
& -p_{i} \mathbf{M}_{i}^{-1}\left(\mathbf{q}_{i}\right) \sum_{j \in \mathcal{N}_{i}} a_{i j} \int_{t-T_{j i}(t)}^{t} \dot{\mathbf{q}}_{j}(\sigma) d \sigma,
\end{aligned}
$$

where we defined the relative position errors

$$
\mathbf{e}_{i}:=\sum_{j \in \mathcal{N}_{i}} a_{i j}\left(\mathbf{q}_{i}-\mathbf{q}_{j}\right), \quad i \leq N
$$

and we used the identity

$$
\int_{t-T_{j i}(t)}^{t} \dot{\mathbf{q}}_{j}(\sigma) d \sigma=\mathbf{q}_{j}-\mathbf{q}_{j}\left(t-T_{j i}(t)\right)
$$


Next, we proceed to construct a Lyapunov-Krassovskiu functional. First, as in [17], we introduce the energy-like function $V_{i}: \mathbb{R}^{2 n} \rightarrow \mathbb{R}_{\geq 0}$, defined as

$$
V_{i}\left(\mathbf{q}_{i}, \dot{\mathbf{q}}_{i}\right)=\frac{1}{2 p_{i}} \dot{\mathbf{q}}_{i}^{\top} \mathbf{M}_{i}\left(\mathbf{q}_{i}\right) \dot{\mathbf{q}}_{i}+\frac{1}{4} \sum_{j \in \mathcal{N}_{i}} a_{i j}\left|\mathbf{q}_{i}-\mathbf{q}_{j}\right|^{2}
$$

Also, using (13) we find that the derivative of $V_{i}$ along the trajectories of (11) yields

$$
\begin{aligned}
\dot{V}_{i}= & -\frac{d_{i}}{p_{i}}\left|\dot{\mathbf{q}}_{i}\right|^{2}-\sum_{j \in \mathcal{N}_{i}} a_{i j} \dot{\mathbf{q}}_{i}^{\top} \int_{t-T_{j i}(t)}^{t} \dot{\mathbf{q}}_{j}(\sigma) d \sigma \\
& -\frac{1}{2} \sum_{j \in \mathcal{N}_{i}} a_{i j}\left(\dot{\mathbf{q}}_{i}+\dot{\mathbf{q}}_{j}\right)^{\top}\left(\mathbf{q}_{i}-\mathbf{q}_{j}\right) .
\end{aligned}
$$

Furthermore, using successively Young's and CauchySchwartz inequalities we obtain that, for any $\alpha_{i}>0$,

$$
\begin{aligned}
\dot{\mathbf{q}}_{i}^{\top} \int_{t-T_{j i}(t)}^{t} \dot{\mathbf{q}}_{j}(\sigma) d \sigma & \leq \frac{\alpha_{i}}{2}\left|\dot{\mathbf{q}}_{i}\right|^{2}+\frac{1}{2 \alpha_{i}}\left|\int_{t-T_{j i}(t)}^{t} \dot{\mathbf{q}}_{j}(\sigma) d \sigma\right|^{2} \\
& \leq \frac{\alpha_{i}}{2}\left|\dot{\mathbf{q}}_{i}\right|^{2}+\frac{{ }^{*} T_{j i}}{2 \alpha_{i}} \int_{t-* T_{j i}}^{t}\left|\dot{\mathbf{q}}_{j}(\sigma)\right|^{2} d \sigma
\end{aligned}
$$

hence,

$$
\begin{aligned}
\dot{V}_{i} \leq & \sum_{j \in \mathcal{N}_{i}} a_{i j}\left[\frac{\alpha_{i}}{2}\left|\dot{\mathbf{q}}_{i}\right|^{2}+\frac{{ }^{*} T_{j i}}{2 \alpha_{i}} \int_{t-* T_{j i}}^{t}\left|\dot{\mathbf{q}}_{j}(\sigma)\right|^{2} d \sigma\right] \\
& -\frac{d_{i}}{p_{i}}\left|\dot{\mathbf{q}}_{i}\right|^{2}-\frac{1}{2} \sum_{j \in \mathcal{N}_{i}} a_{i j}\left(\dot{\mathbf{q}}_{i}+\dot{\mathbf{q}}_{j}\right)^{\top}\left(\mathbf{q}_{i}-\mathbf{q}_{j}\right) .
\end{aligned}
$$

Next, for each $i \leq N$ let the given constants on the right hand side of $(10)$ generate $d_{i}$ satisfying this condition. Then, there exists $\delta_{i}>0$ such that

$$
2 d_{i}>p_{i} \sum_{j \in \mathcal{N}_{i}} a_{i j}\left(\alpha_{i}+\frac{{ }^{*} T_{j i}^{2}}{\alpha_{j}}+\frac{{ }^{*} T_{i j} \delta_{i}}{2}\right) .
$$

Consider next the functional $W_{i}: \mathcal{C}[-h, 0]^{n} \rightarrow \mathbb{R}_{\geq 0}$ defined as

$$
W_{i}\left(\dot{\mathbf{q}}_{t}\right):=\left(\frac{{ }^{*} T_{j i}}{2 \alpha_{i}}+\delta_{i}\right) \sum_{j \in \mathcal{N}_{i}} a_{i j} \int_{-{ }^{*} T_{j i}}^{0} \int_{t+\sigma}^{t}\left|\dot{\mathbf{q}}_{j}(\theta)\right|^{2} d \theta d \sigma
$$

where $\dot{\mathbf{q}}_{t}(\theta):=\dot{\mathbf{q}}(t+\theta)$. We note that $W_{i}\left(\dot{\mathbf{q}}_{t}\right) \geq 0$ and there exists $c_{i}>0$ such that $W_{i}\left(\dot{\mathbf{q}}_{t}\right) \leq c_{i}\left\|\dot{\mathbf{q}}_{t}\right\|^{2}$-see (1). The total derivative of $\dot{W}_{i}$ yields

$\dot{W}_{i}=\left[\frac{{ }^{*} T_{j i}}{2 \alpha_{i}}+\delta_{i}\right] \sum_{j \in \mathcal{N}_{i}} a_{i j}\left[{ }^{*} T_{j i}\left|\dot{\mathbf{q}}_{j}(t)\right|^{2}-\int_{t-* T_{j i}}^{t}\left|\dot{\mathbf{q}}_{j}(\sigma)\right|^{2} d \sigma\right]$

Next, defining $E_{i}\left(\mathbf{q}_{i}, \dot{\mathbf{q}}_{i}, \dot{\mathbf{q}}_{t}\right):=V_{i}\left(\mathbf{q}_{i}, \dot{\mathbf{q}}_{i}\right)+W_{i}\left(\dot{\mathbf{q}}_{t}\right)-c f$.
$[29,30]$, we obtain

$$
\begin{aligned}
\dot{E}_{i} \leq & -\frac{d_{i}}{p_{i}}\left|\dot{\mathbf{q}}_{i}\right|^{2}+\sum_{j \in \mathcal{N}_{i}} a_{i j}\left[\frac{\alpha_{i}}{2}\left|\dot{\mathbf{q}}_{i}\right|^{2}+\left(\frac{{ }^{*} T_{j i}^{2}}{2 \alpha_{i}}+\delta_{i}{ }^{*} T_{j i}\right)\left|\dot{\mathbf{q}}_{j}\right|^{2}\right] \\
& -\delta_{i} \sum_{j \in \mathcal{N}_{i}} a_{i j} \int_{t-{ }^{*} T_{j i}}^{t}\left|\dot{\mathbf{q}}_{j}(\sigma)\right|^{2} d \sigma \\
& -\frac{1}{2} \sum_{j \in \mathcal{N}_{i}} a_{i j}\left(\dot{\mathbf{q}}_{i}+\dot{\mathbf{q}}_{j}\right)^{\top}\left(\mathbf{q}_{i}-\mathbf{q}_{j}\right)
\end{aligned}
$$

and, defining $E\left(\mathbf{q}, \dot{\mathbf{q}}, \dot{\mathbf{q}}_{t}\right):=\sum_{i \in \bar{N}} E_{i}\left(\mathbf{q}_{i}, \dot{\mathbf{q}}_{i}, \dot{\mathbf{q}}_{t}\right)$, we obtain

$$
\begin{aligned}
\dot{E} \leq & -\sum_{i \in \bar{N}} \sum_{j \in \mathcal{N}_{i}} a_{i j}\left[\left(\frac{d_{i}}{p_{i} L_{i i}}-\frac{\alpha_{i}}{2}\right)\left|\dot{\mathbf{q}}_{i}\right|^{2}\right. \\
& \left.-\left(\frac{{ }^{*} T_{j i}^{2}}{2 \alpha_{i}}+\delta_{i}{ }^{*} T_{j i}\right)\left|\dot{\mathbf{q}}_{j}\right|^{2}\right] \\
& -\sum_{i \in \bar{N}} \delta_{i} \sum_{j \in \mathcal{N}_{i}} a_{i j} \int_{t-{ }^{*} T_{j i}}^{t}\left|\dot{\mathbf{q}}_{j}(\sigma)\right|^{2} d \sigma
\end{aligned}
$$

where $L_{i i}$ is the diagonal element of the Laplacian, defined as $L_{i i}:=\sum_{j \in \mathcal{N}_{i}} a_{i j}$, and we used the fact that the Laplacian matrix is symmetric, so

$$
\sum_{i \in \bar{N}} \sum_{j \in \mathcal{N}_{i}} a_{i j}\left(\dot{\mathbf{q}}_{i}+\dot{\mathbf{q}}_{j}\right)^{\top}\left(\mathbf{q}_{i}-\mathbf{q}_{j}\right)=0
$$

Now, as in [17], to compact the notation, we introduce $\mathbf{Q}:=\left[\left|\dot{\mathbf{q}}_{1}\right|^{2} \cdots\left|\dot{\mathbf{q}}_{N}\right|^{2}\right]^{\top}$ and

$\Psi=\left[\begin{array}{ccc}\frac{\alpha_{1}}{2}-\frac{d_{1}}{p_{1} L_{11}} & \ldots & \left(\frac{* T_{N 1}}{2 \alpha_{1}}+\delta_{1}\right){ }^{*} T_{N 1} a_{1 N} \\ \vdots & \ddots & \vdots \\ \left(\frac{{ }^{*} T_{1 N}}{2 \alpha_{N}}+\delta_{N}\right){ }^{*} T_{1 N} a_{N 1} & \ldots & \frac{\alpha_{N}}{2}-\frac{d_{N}}{p_{N} L_{N N}}\end{array}\right]$,

so

$$
\dot{E} \leq \mathbf{1}_{N}^{\top} \mathbf{\Psi} \mathbf{Q}-\sum_{i \in \bar{N}} \delta_{i} \sum_{j \in \mathcal{N}_{i}} a_{i j} \int_{t-* T_{j i}}^{t}\left|\dot{\mathbf{q}}_{j}(\sigma)\right|^{2} d \sigma .
$$

In view of (10), for each $i \leq N$, the numbers

$$
\lambda_{i}:=\frac{d_{i}}{p_{i} L_{i i}}-\frac{\alpha_{i}}{2}-\sum_{j \in \mathcal{N}_{i}} a_{i j}\left(\frac{{ }^{*} T_{i j}}{2 \alpha_{j}}+\delta_{i}\right){ }^{*} T_{i j}
$$

are positive and, consequently, $-\boldsymbol{\Psi}$ is diagonal dominant. Therefore,

$$
\dot{E} \leq-\sum_{i \in \bar{N}}\left[\lambda_{i}\left|\dot{\mathbf{q}}_{i}\right|^{2}+\delta_{i} \sum_{j \in \mathcal{N}_{i}} a_{i j} \int_{t-* T_{j i}}^{t}\left|\dot{\mathbf{q}}_{j}(\sigma)\right|^{2} d \sigma\right]
$$

that is, $\dot{E}$ is negative semi-definite. In order to render it negative definite we introduce the cross term

$$
U(\mathbf{e}, \dot{\mathbf{q}}):=\mathbf{h}(\mathbf{e})^{\top} \mathbf{P}^{-1} \mathbf{M}(\mathbf{q}) \dot{\mathbf{q}}
$$


where $\quad \mathbf{P} \quad:=\operatorname{diag}\left[p_{i}\right] \otimes \mathbf{I}_{n} \quad \in \quad \mathbb{R}^{N n \times N n}$; $\mathbf{M}(\mathbf{q}):=\operatorname{blockdiag}\left[\mathbf{M}_{i}\left(\mathbf{q}_{i}\right)\right]$ and

$$
\mathbf{h}(\mathbf{e}):=\frac{1}{1+|\mathbf{e}|} \mathbf{e}, \quad \mathbf{e}:=\left[\mathbf{e}_{1}^{\top} \cdots \mathbf{e}_{N}^{\top}\right]^{\top} .
$$

Remark 4. For further development, we observe that $\mathbf{h}$ satisfies the following: i) $|\mathbf{h}(\mathbf{e})| \leq 1$, ii) $|\mathbf{h}(\mathbf{e})| \leq|\mathbf{e}|$, and iii) $|\dot{\mathbf{h}}(\mathbf{e})| \leq 2|\dot{\mathbf{e}}|$. Furthermore, from the fact that $\dot{\mathbf{e}}=$ $\left(\mathbf{L} \otimes \mathbf{I}_{n}\right) \dot{\mathbf{q}}$, it holds that $|\dot{\mathbf{h}}| \leq 2 \lambda_{M}\{\mathbf{L}\}|\dot{\mathbf{q}}|$.

We are now ready to present our Lyapunov-Krasovskiu functional candidate. Let $\mathcal{V}: \mathbb{R}_{\geq 0} \times \mathcal{C}\left[-{ }^{*} T, 0\right] \rightarrow \mathbb{R}_{\geq 0}$, with ${ }^{*} T:=\min \left\{{ }^{*} T_{i j}\right\}$, be defined as

$$
\mathcal{V}\left(t, x_{t}\right)=E\left(\mathbf{q}(t), \dot{\mathbf{q}}(t), \dot{\mathbf{q}}_{t}\right)+\epsilon U(\mathbf{e}(t), \dot{\mathbf{q}}(t))
$$

where $x_{t}:=\left[\mathbf{e}(t)^{\top} \dot{\mathbf{q}}(t)^{\top} \dot{\mathbf{q}}_{t}^{\top}\right]^{\top}$ and $\epsilon>0$. We show that $\mathcal{V}$ verifies the conditions of Theorem 1 . To that end, we stress that $U(\mathbf{e}, \dot{\mathbf{q}})$ can be also written as

$$
U(\mathbf{e}, \dot{\mathbf{q}})=\sum_{i \in \bar{N}} \frac{1}{p_{i}} \mathbf{h}_{i}(\mathbf{e})^{\top} \mathbf{M}_{i}\left(\mathbf{q}_{i}\right) \dot{\mathbf{q}}_{i},
$$

with $\mathbf{h}_{i}(\mathbf{e})=\frac{1}{1+|\mathbf{e}|} \mathbf{e}_{i}$. Therefore,

$$
\begin{array}{r}
E\left(\mathbf{q}, \dot{\mathbf{q}}, \dot{\mathbf{q}}_{t}\right)+\epsilon U(\mathbf{e}, \dot{\mathbf{q}})=\sum_{i \in \bar{N}} W_{i}+\frac{1}{4} \sum_{i \in \bar{N}} \sum_{j \in \mathcal{N}_{i}} a_{i j}\left|\mathbf{q}_{i}-\mathbf{q}_{j}\right|^{2} \\
+\sum_{i \in \bar{N}} \frac{1}{2 p_{i}}\left[\left[\dot{\mathbf{q}}_{i}+\epsilon \mathbf{h}_{i}\right]^{\top} \mathbf{M}_{i}\left[\dot{\mathbf{q}}_{i}+\epsilon \mathbf{h}_{i}\right]-\epsilon^{2} \mathbf{h}_{i}^{\top} \mathbf{M}_{i} \mathbf{h}_{i}\right]
\end{array}
$$

and the term $\mathbf{h}_{i}^{\top} \mathbf{M}_{i} \mathbf{h}_{i}=\frac{1}{(1+|\mathbf{e}|)^{2}} \mathbf{e}_{i}^{\top} \mathbf{M}_{i} \mathbf{e}_{i}$ admits the bound

$$
\begin{aligned}
\frac{1}{p_{i}} \mathbf{h}_{i}^{\top} \mathbf{M}_{i} \mathbf{h}_{i} & \leq \frac{m_{2 i}}{p_{i}}\left|\sum_{j \in \mathcal{N}_{i}} a_{i j}\left(\mathbf{q}_{i}-\mathbf{q}_{j}\right)\right|^{2} \\
& \leq \bar{m} \bar{L} \sum_{j \in \mathcal{N}_{i}} a_{i j}\left|\mathbf{q}_{i}-\mathbf{q}_{j}\right|^{2}
\end{aligned}
$$

where $\bar{m}:=\max _{i \in \bar{N}}\left\{\frac{m_{2 i}}{p_{i}}\right\}$ and $\bar{L}:=\max _{i \in \bar{N}}\left\{L_{i i}\right\}$. Therefore, since $c_{i}\left\|\dot{\mathbf{q}}_{t}\right\|^{2} \geq W_{i} \geq 0$ for all $i \leq \bar{N}$, by setting

$$
\epsilon<\sqrt{\frac{1}{2 \bar{m} \bar{L}}}
$$

we find that $\mathcal{V}$ satisfies (3) with $\beta_{1}(s), \beta_{2}(s) \propto s^{2}$.

We compute next the total derivative of $E+\epsilon U$. To that end, we write

$$
\dot{U}=\dot{\mathbf{h}}^{\top} \mathbf{P}^{-1} \mathbf{M} \dot{\mathbf{q}}+\mathbf{h}^{\top} \mathbf{P}^{-1}[\dot{\mathbf{M}} \dot{\mathbf{q}}+\mathbf{M} \ddot{\mathbf{q}}]
$$

and we observe that

$$
\dot{\mathbf{h}}^{\top} \mathbf{P}^{-1} \mathbf{M} \dot{\mathbf{q}} \leq 2 \bar{m} \lambda_{M}\{\mathbf{L}\}|\dot{\mathbf{q}}|^{2}
$$

Then, using $\mathbf{P} 1$ and (11), the last terms of $\dot{U}$ can be written as

$$
\begin{aligned}
\mathbf{h}^{\top} \mathbf{P}^{-1}[\dot{\mathbf{M}} \dot{\mathbf{q}} & +\mathbf{M} \ddot{\mathbf{q}}]= \\
& \sum_{i \in \bar{N}} \frac{1}{p_{i}} \mathbf{h}_{i}^{\top}\left[\mathbf{C}_{i}^{\top} \dot{\mathbf{q}}_{i}-d_{i} \dot{\mathbf{q}}_{i}-p_{i} \mathbf{e}_{i}\right] \\
& -\sum_{i \in \bar{N}} \mathbf{h}_{i}^{\top} \sum_{j \in \mathcal{N}_{i}} a_{i j} \int_{t-T_{j i}(t)}^{t} \dot{\mathbf{q}}_{j}(\sigma) d \sigma .
\end{aligned}
$$

On the other hand, in view of $\mathbf{P 2}$ and the boundedness of $\mathbf{h}$, we have $\mathbf{h}_{i}^{\top} \mathbf{C}_{i}^{\top}\left(\mathbf{q}_{i}, \dot{\mathbf{q}}_{i}\right) \dot{\mathbf{q}}_{i} \leq k_{c i}\left|\dot{\mathbf{q}}_{i}\right|^{2}$. In addition, invoking Young's inequality for any $\mu>0$, we obtain

$$
-\mathbf{h}_{i}^{\top} \dot{\mathbf{q}}_{i} \leq \frac{\mu}{2}\left|\mathbf{h}_{i}\right|^{2}+\frac{1}{2 \mu}\left|\dot{\mathbf{q}}_{i}\right|^{2}
$$

and

$$
\begin{aligned}
\mathbf{h}_{i}^{\top} \sum_{j \in \mathcal{N}_{i}} a_{i j} & \int_{t-T_{j i}(t)}^{t} \dot{\mathbf{q}}_{j}(\sigma) d \sigma \\
& \leq \frac{\mu}{2}\left|\mathbf{h}_{i}\right|^{2}+\frac{1}{2 \mu}\left|\sum_{j \in \mathcal{N}_{i}} a_{i j} \int_{t-T_{j i}(t)}^{t} \dot{\mathbf{q}}_{j}(\sigma) d \sigma\right|^{2}, \\
& \leq \frac{\mu}{2}\left|\mathbf{h}_{i}\right|^{2}+\frac{L_{i i}}{2 \mu} \sum_{j \in \mathcal{N}_{i}} a_{i j}{ }^{*} T_{j i} \int_{t-{ }^{*} T_{j i}}^{t}\left|\dot{\mathbf{q}}_{j}(\sigma)\right|^{2} d \sigma
\end{aligned}
$$

where the last inequality follows from the fact that $\left(\sum a b\right)^{2} \leq \sum a^{2} \sum b^{2}$ and the Cauchy-Schwartz inequality. Moreover,

$$
\left|\mathbf{h}_{i}\right|^{2} \leq \frac{1}{1+|\mathbf{e}|}\left|\mathbf{e}_{i}\right|^{2}
$$

so $\dot{U}$ satisfies

$$
\begin{aligned}
\dot{U} \leq & \sum_{i \in \bar{N}}\left(2 \bar{m} \lambda_{M}\{\mathbf{L}\}+\frac{k_{c i}}{p_{i}}+\frac{d_{i}}{2 \mu p_{i}}\right)\left|\dot{\mathbf{q}}_{i}\right|^{2} \\
& +\sum_{i \in \bar{N}}\left(\frac{\mu d_{i}}{2 p_{i}}+\frac{\mu}{2}-1\right) \frac{1}{1+|\mathbf{e}|}\left|\mathbf{e}_{i}\right|^{2} \\
& +\sum_{i \in \bar{N}}\left[\frac{L_{i i}}{2 \mu} \sum_{j \in \mathcal{N}_{i}} a_{i j}{ }^{*} T_{j i} \int_{t-* T_{j i}}^{t}\left|\dot{\mathbf{q}}_{j}(\sigma)\right|^{2} d \sigma\right] .
\end{aligned}
$$

and, in turn, it holds that

$$
\begin{aligned}
\dot{\mathcal{V}} \leq & -\sum_{i \in \bar{N}}\left(\lambda_{i}-\epsilon\left(2 \bar{m} \lambda_{M}\{\mathbf{L}\}+\frac{k_{c i}}{p_{i}}+\frac{d_{i}}{2 \mu p_{i}}\right)\right)\left|\dot{\mathbf{q}}_{i}(t)\right|^{2} \\
& -\epsilon \sum_{i \in \bar{N}}\left(1-\frac{\mu d_{i}}{2 p_{i}}-\frac{\mu}{2}\right) \frac{1}{1+|\mathbf{e}|}\left|\mathbf{e}_{i}(t)\right|^{2} \\
& -\sum_{i \in \bar{N}}\left[\sum_{j \in \mathcal{N}_{i}} a_{i j}\left(\delta_{i}-\frac{L_{i i}{ }^{*} T_{j i}}{2 \mu} \epsilon\right) \int_{t-{ }^{*} T_{j i}}^{t}\left|\dot{\mathbf{q}}_{j}(\sigma)\right|^{2} d \sigma\right]
\end{aligned}
$$


and, defining $\underline{\lambda}:=\min _{i \in \bar{N}}\left\{\lambda_{i}\right\}, \underline{\delta}:=\min _{i \in \bar{N}}\left\{\delta_{i}\right\}, \bar{k}_{c}:=\max _{i \in \bar{N}}\left\{\frac{k_{c i}}{p_{i}}\right\}$, $\bar{d}:=\max _{i \in \bar{N}}\left\{\frac{d_{i}}{p_{i}}\right\}$, and ${ }^{*} T:=\max _{\substack{i \in N \\ j \in \mathcal{N}_{i}}}\left\{{ }^{*} T_{j i}\right\}$ we obtain

$$
\begin{aligned}
\dot{\mathcal{V}} \leq- & \mu_{1}|\dot{\mathbf{q}}(t)|^{2}-\frac{\epsilon \mu_{2}}{1+|\mathbf{e}(t)|}|\mathbf{e}(t)|^{2} \\
& -\mu_{3} \sum_{i \in \bar{N}} \sum_{j \in \mathcal{N}_{i}} a_{i j} \int_{t-{ }^{*} T_{j i}}^{t}\left|\dot{\mathbf{q}}_{j}(\sigma)\right|^{2} d \sigma,
\end{aligned}
$$

where $\mu_{1}:=\underline{\lambda}-\epsilon\left(2 \bar{m} \lambda_{M}\{\mathbf{L}\}+\bar{k}_{c}+\frac{\bar{d}}{2 \mu}\right), \mu_{2}:=1-$ $\frac{\mu}{2}(\bar{d}+1)$, and $\mu_{3}:=\underline{\delta}-\frac{\bar{L}^{*} T}{2 \mu} \epsilon$. Thus, setting

$$
\epsilon<\min \left\{\frac{\underline{\lambda}}{2 \bar{m} \lambda_{M}\{\mathbf{L}\}+\bar{k}_{c}+\frac{\bar{d}}{2 \mu}}, \frac{2 \mu \underline{\delta}}{\bar{L} T^{*}}, \sqrt{\frac{1}{2 \bar{m} \bar{L}}}\right\},
$$

for any $\mu$ satisfying $\mu<\frac{2}{d+1}$, ensures that $\dot{\mathcal{V}}$ satisfies (4). The first statement follows invoking Theorem 1.

Next, let $\mathbf{u}_{i}$ be an additive input to the system (6), which defines a passive map $\mathbf{u}_{i}+\boldsymbol{\tau}_{i}$. Therefore, after the previous computations we obtain

$$
\begin{aligned}
\dot{\mathcal{V}} \leq- & \mu_{1}|\dot{\mathbf{q}}(t)|^{2}-\frac{\epsilon \mu_{2}}{1+|\mathbf{e}(t)|}|\mathbf{e}(t)|^{2} \\
& +\sum_{i \in \bar{N}} \frac{1}{p_{i}}\left[\dot{\mathbf{q}}_{i}(t)+\epsilon \mathbf{h}_{i}\left(\mathbf{e}_{i}(t)\right)\right]^{\top} \mathbf{u}_{i}(t) .
\end{aligned}
$$

So, using the triangle inequality and (19) we obtain

$\dot{\mathcal{V}} \leq-\frac{\mu_{1}}{2}|\dot{\mathbf{q}}(t)|^{2}-\frac{1}{2} \frac{\epsilon \mu_{2}}{1+|\mathbf{e}(t)|}|\mathbf{e}(t)|^{2}+\frac{1}{2 \underline{p}^{2}}\left[\frac{1}{\mu_{1}}+\frac{\epsilon}{\mu_{2}}\right]|\mathbf{u}(t)|^{2}$

where $\underline{p}:=\min \left\{p_{i}\right\}$. Therefore, the system is Input-toState stable with respect to $\mathbf{u}$.

\section{Leader-Follower Consensus}

We speak of leader-follower consensus if, given an agreement position $\mathbf{q}_{\ell} \in \mathbb{R}^{n}$ which is accessible to at least one follower (but not necessarily to all of them)

$$
\lim _{t \rightarrow \infty}\left|\dot{\mathbf{q}}_{i}(t)\right|=0, \quad \lim _{t \rightarrow \infty} \mathbf{q}_{i}(t)=\mathbf{q}_{\ell}
$$

for all $i \in[0, N]$.

Proposition 2. Consider the system (6) in closed-loop with

$$
\begin{aligned}
\tau_{i}= & \nabla_{\mathbf{q}_{i}} \mathcal{U}_{i}\left(\mathbf{q}_{i}\right)-d_{i} \dot{\mathbf{q}}_{i}-p_{i} a_{i \ell}\left(\mathbf{q}_{i}-\mathbf{q}_{\ell}\right) \\
& -p_{i} \sum_{j \in \mathcal{N}_{i}} a_{i j}\left(\mathbf{q}_{i}-\mathbf{q}_{j}\left(t-T_{j i}(t)\right)\right)
\end{aligned}
$$

for each $i \leq N$ and let (10) hold and under Assumptions A1-A3. Then, the equilibrium $(\dot{\mathbf{q}}, \mathbf{q})=\left(\mathbf{0}, \mathbf{1}_{N} \otimes \mathbf{q}_{\ell}\right)$ is $U G A S$ provided that the damping gain satisfies (10). Furthermore, the the closed-loop system (11) is ISS with respect to additive inputs $\mathbf{u}_{i} \in \mathbb{R}^{n}$.
Proof. The proof follows along the same lines as that of Proposition 1. Let $\mathcal{N}_{i \ell}:=\mathcal{N}_{i} \cup\{j=\ell\}$. We (re)define the relative position errors (12) as

$$
\begin{aligned}
\mathbf{e}_{i} & :=a_{i \ell}\left(\mathbf{q}_{i}-\mathbf{q}_{\ell}\right)+\sum_{j \in \mathcal{N}_{i}} a_{i j}\left(\mathbf{q}_{i}-\mathbf{q}_{j}\right) \\
& =\sum_{j \in \mathcal{N}_{i \ell}} a_{i j}\left(\mathbf{q}_{i}-\mathbf{q}_{j}\right) .
\end{aligned}
$$

Then, the closed-loop equations (6), (23) are given by (11) with $\mathcal{N}_{i}$ replaced by $\mathcal{N}_{i \ell}$. Next, we introduce

$$
\begin{aligned}
H\left(\mathbf{q}, \dot{\mathbf{q}}, \dot{\mathbf{q}}_{t}\right)= & \frac{1}{4} \sum_{i \in \bar{N}}\left[\frac{2}{p_{i}} \dot{\mathbf{q}}_{i}^{\top} \mathbf{M}_{i} \dot{\mathbf{q}}_{i}+a_{i \ell}\left|\mathbf{q}_{i}-\mathbf{q}_{\ell}\right|^{2}\right] \\
& +\sum_{i \in \bar{N}}\left[\frac{1}{4} \sum_{j \in \mathcal{N}_{i \ell}} a_{i j}\left|\mathbf{q}_{i}-\mathbf{q}_{j}\right|^{2}+W_{i}\left(\dot{\mathbf{q}}_{t}\right)\right],
\end{aligned}
$$

where $W_{i}\left(\dot{\mathbf{q}}_{t}\right)$ is redefined upon (15) by replacing $\mathcal{N}_{i}$ with $\mathcal{N}_{i \ell}$. Akin to $\dot{E}$, im view of (14), the total derivative of $H$ along the closed-loop trajectories satisfies

$$
\dot{H} \leq-\sum_{i \in \bar{N}}\left[\lambda_{i}\left|\dot{\mathbf{q}}_{i}\right|^{2}+\delta_{i} \sum_{j \in \mathcal{N}_{i \ell}} a_{i j} \int_{t-* T_{j i}}^{t}\left|\dot{\mathbf{q}}_{j}(\sigma)\right|^{2} d \sigma\right] .
$$

Thus, we introduce the Lyapunov-Krasovskii functional

$$
\mathcal{V}\left(t, x_{t}\right)=H\left(\mathbf{q}(t), \dot{\mathbf{q}}(t), \dot{\mathbf{q}}_{t}\right)+\epsilon U(\mathbf{e}(t), \dot{\mathbf{q}}(t)),
$$

where $U(\mathbf{e}, \dot{\mathbf{q}})$ is defined in (17). Note that

$$
\begin{aligned}
& H\left(\mathbf{q}, \dot{\mathbf{q}}, \dot{\mathbf{q}}_{t}\right)+\epsilon U(\mathbf{e}, \dot{\mathbf{q}})= \\
& \sum_{i \in \bar{N}}\left[\frac{1}{2 p_{i}}\left[\dot{\mathbf{q}}_{i}+\epsilon \mathbf{h}_{i}\right]^{\top} \mathbf{M}_{i}\left[\dot{\mathbf{q}}_{i}+\epsilon \mathbf{h}_{i}\right]+\frac{a_{i \ell}}{4}\left|\mathbf{q}_{i}-\mathbf{q}_{\ell}\right|^{2}\right] \\
& \quad+\sum_{i \in \bar{N}} \sum_{j \in \mathcal{N}_{i \ell}} a_{i j}\left(\frac{{ }^{*} T_{j i}}{2 \alpha_{i}}+\delta_{i}\right) \int_{-* T_{j i}}^{0} \int_{t+\sigma}^{t}\left|\dot{\mathbf{q}}_{j}(\theta)\right|^{2} d \theta d \sigma \\
& \quad+\frac{1}{4} \sum_{i \in \bar{N}}\left[\sum_{j \in \mathcal{N}_{i \ell}} a_{i j}\left|\mathbf{q}_{i}-\mathbf{q}_{j}\right|^{2}-\frac{2 \epsilon^{2}}{p_{i}} \mathbf{h}_{i}^{\top} \mathbf{M}_{i} \mathbf{h}_{i}\right]
\end{aligned}
$$

Since

$$
\frac{1}{p_{i}} \mathbf{h}_{i}^{\top} \mathbf{M}_{i}\left(\mathbf{q}_{i}\right) \mathbf{h}_{i} \leq \bar{m} \bar{L}_{\ell} \sum_{j \in \mathcal{N}_{i \ell}} a_{i j}\left|\mathbf{q}_{i}-\mathbf{q}_{j}\right|^{2},
$$

where $\bar{L}_{\ell}:=\max _{i \in \bar{N}}\left\{L_{i i}+a_{i \ell}\right\}$, setting $\epsilon<\sqrt{\frac{1}{\bar{m} \bar{L}_{\ell}}}$ ensures that $H\left(\mathbf{q}, \dot{\mathbf{q}}, \dot{\mathbf{q}}_{t}\right)+\epsilon U(\mathbf{e}, \dot{\mathbf{q}})$ is positive definite and radially unbounded with regards to $\mathbf{e}$ and $\dot{\mathbf{q}}$.

Proceeding as in the Leaderless consensus case, we can show that

$$
\mathcal{V}\left(t, x_{t}\right)=H\left(\mathbf{q}(t), \dot{\mathbf{q}}(t), \dot{\mathbf{q}}_{t}\right)+\epsilon U(\mathbf{e}(t), \dot{\mathbf{q}}(t))
$$

satisfies

$$
\begin{aligned}
\dot{\mathcal{V}} \leq- & \phi_{1}|\dot{\mathbf{q}}(t)|^{2}-\frac{\epsilon \beta_{2}}{1+|\mathbf{e}(t)|}|\mathbf{e}(t)|^{2} \\
& -\phi_{3} \sum_{i \in \bar{N}} \sum_{j \in \mathcal{N}_{i \ell}} a_{i j} \int_{t-* T_{j i}}^{t}\left|\dot{\mathbf{q}}_{j}(\sigma)\right|^{2} d \sigma
\end{aligned}
$$


where $\phi_{1}:=\underline{\lambda}-\epsilon\left(2 \bar{m} \lambda_{M}\left\{\mathbf{L}_{\ell}\right\}+\bar{k}_{c}+\frac{\bar{d}}{2 \mu}\right) ;$ and $\phi_{3}:=\underline{\delta}-$ $\frac{\bar{L}_{\ell}^{*} T}{2 \mu} \epsilon$. Thus, setting

$$
\epsilon<\min \left\{\frac{\underline{\lambda}}{2 \bar{m} \lambda_{M}\left\{\mathbf{L}_{\ell}\right\}+\bar{k}_{c}+\frac{\bar{d}}{2 \mu}}, \frac{2 \mu \underline{\delta}}{\bar{L}_{\ell}{ }^{*} T}, \sqrt{\frac{1}{2 \bar{m} \bar{L}_{\ell}}}\right\},
$$

for any $\mu$ satisfying $\mu<\frac{2}{d+1}$, ensures that $\dot{\mathcal{V}}$ is negative definite so, after Theorem 1 , the result follows. The statement on Input-to-State stability follows mutatis mutandis as for Proposition 1.

Remark 5. Suppose that velocity measurements are not available, but that a velocity estimate $\hat{\dot{\mathbf{q}}}_{i}$ is available from a velocity observer. In this case, the certainty-equivalent controllers (9) and (23) become

$$
\tau_{i}=\nabla_{\mathbf{q}_{i}} \mathcal{U}_{i}\left(\mathbf{q}_{i}\right)-d_{i} \hat{\dot{\mathbf{q}}}_{i}-p_{i} \sum_{j \in \mathcal{N}_{i}} a_{i j}\left(\mathbf{q}_{i}-\mathbf{q}_{j}\left(t-T_{j i}(t)\right)\right)
$$

and

$$
\begin{aligned}
\tau_{i}= & \nabla_{\mathbf{q}_{i}} \mathcal{U}_{i}\left(\mathbf{q}_{i}\right)-d_{i} \hat{\mathbf{q}}_{i}-p_{i} a_{i \ell}\left(\mathbf{q}_{i}-\mathbf{q}_{\ell}\right) \\
& -p_{i} \sum_{j \in \mathcal{N}_{i}} a_{i j}\left(\mathbf{q}_{i}-\mathbf{q}_{j}\left(t-T_{j i}(t)\right)\right),
\end{aligned}
$$

respectively. Correspondingly, the closed-loop system (11) becomes

$$
\begin{aligned}
\ddot{\mathbf{q}}_{i}= & -\mathbf{M}_{i}^{-1}\left(\mathbf{q}_{i}\right)\left[\mathbf{C}_{i}\left(\mathbf{q}_{i}, \dot{\mathbf{q}}_{i}\right) \dot{\mathbf{q}}_{i}+d_{i} \dot{\mathbf{q}}_{i}+p_{i} \mathbf{e}_{i}\right] \\
& -p_{i} \mathbf{M}_{i}^{-1}\left(\mathbf{q}_{i}\right) \sum_{j \in \mathcal{N}_{i}} a_{i j} \int_{t-T_{j i}(t)}^{t} \dot{\mathbf{q}}_{j}(\sigma) d \sigma \\
& +d_{i} \mathbf{M}_{i}^{-1}\left(\mathbf{q}_{i}\right) \tilde{\dot{\mathbf{q}}}_{i},
\end{aligned}
$$

where $\tilde{\mathbf{q}}_{i}:=\dot{\mathbf{q}}_{i}-\hat{\dot{\mathbf{q}}}_{i}$ is the velocity estimation error; similarly for the closed-loop system with the controller (26) in which case $\mathcal{N}_{i}$ is replaced with $\mathcal{N}_{i \ell}$. In either case, (21) holds with $\mathbf{u}(t)=\tilde{\dot{\mathbf{q}}}(t)$ so, under the hypothesis that the latter converges asymptotically to zero, so do $\dot{\mathbf{q}}(t)$ and $\dot{\mathbf{e}}(t)$. If furthermore, the origin for estimation errors dynamics is uniformly globally asymptotically stable, so is the null solution for the overall closed-loop dynamics.

\section{Conclusions}

We propose a novel Strict Lyapunov-Krasovskiü Functional for the leaderless and leader-follower consensus in networks of EL-agents controlled by simple decentralized $\mathrm{P}+\mathrm{d}$ schemes for which the communications impose variable time-delays that may not be differentiable. We prove that the agents positions and velocities globally, uniformly and asymptotically converge to a consensus position and to zero, respectively. Moreover, by directly replacing the velocity measurements by the velocity estimates obtained from a velocity observer that accepts a SLF, as the I\&I velocity observer, the same UGAS property holds. Finally, we also establish the fact that the resulting closed-loop systems are ISS with regards to external inputs (perturbations).

One future research avenue concerns the inclusion of non-holonomic restrictions in the agents dynamics.

\section{Acknowledgements}

This work is partially supported by the Mexican CONACyT Basic Scientific Research grant CB-282807; by the Dept. STITS of University of Paris Saclay; by the Government of Russian Federation (grant 08-08); and by the ECOS-Nord contract M14M02.

\section{References}

[1] A. Rodriguez-Angeles and H. Nijmeijer. Mutual synchronization of robots via estimated state feedback: A cooperative approach. IEEE Transactions on Control Systems Technology, 12(4):542-554, July 2004.

[2] N. Chopra and M.W. Spong. Advances in Robot Control: From Everyday Physics to Human-Like Movements (Eds.) S. Kawamura and M. Svinin, chapter Passivity-based Control of Multiagent Systems, pages 107-134. Springer, 2016.

[3] S.J. Chung and J.J.E. Slotine. Cooperative robot control and synchronization of Lagrangian systems. In 46th IEEE Conference on Decision and Control (CDC), pages 2504-2509, New Orleans, LA, USA, 2007.

[4] S.J. Chung and J.J. Slotine. Cooperative robot control and concurrent synchronization of Lagrangian systems. IEEE Trans. on Robotics, 25(3):686-700, 2009.

[5] W. Ren. Distributed leaderless consensus algorithms for networked Euler-Lagrange systems. Int. Jour. of Control, 82(11):2137-2149, 2009.

[6] R. Olfati-Saber and R.M. Murray. Consensus problems in networks of agents with switching topology and time-delays. IEEE Transactions on Automatic Control, 49(9):1520-1533, 2004.

[7] Y. Cao and W. Ren. Distributed Coordination of Multi-agent Networks: Emergent Problems, Models, and Issues. SpringerVerlag, 2011.

[8] T. Hatanaka, N. Chopra, M. Fujita, and M.W. Spong. Passivity-Based Control and Estimation in Networked Robotics. Communications and Control Engineering. Springer, 2015.

[9] E. Nuño, R. Ortega, L. Basañez, and D. Hill. Synchronization of networks of nonidentical Euler-Lagrange systems with uncertain parameters and communication delays. IEEE Transactions on Automatic Control, 56(4):935-941, 2011.

[10] Y. Liu and N. Chopra. Controlled synchronization of heterogeneous robotic manipulators in the task space. IEEE Transactions on Robotics, 28(1):268-275, 2012.

[11] Y. Liu, H. Min, S. Wang, L. Ma, and Z. Liu. Consensus for multiple heterogeneous Euler-Lagrange systems with time-delay and jointly connected topologies. Journal of the Franklin Institute, 351(6):3351-3363, June 2014.

[12] H. Wang. Consensus of networked mechanical systems with communication delays: A unified framework. IEEE Transactions on Automatic Control, 59(6):1571-1576, 2014.

[13] E. Nuño. Consensus of Euler-Lagrange systems using only position measurements. IEEE Transactions on Control of Network Systems, 5(1):489-498, 2018.

[14] E. Nuño and R. Ortega. Achieving consensus of Euler-Lagrange agents with interconnecting delays and without velocity measurements via passivity-based control. IEEE Transactions on Control Systems Technology, 26(1):222-232, 2018.

[15] J.R. Klotz, S. Obuz, Z. Kan, and W.E. Dixon. Synchronization of uncertain Euler-Lagrange systems with uncertain timevarying communication delays. IEEE Transactions on Cybernetics, 48(2):807-817, 2018. 
[16] E. Nuño, I. Sarras, E. Panteley, and L. Basañez. Consensus in networks of nonidentical Euler-Lagrange systems with variable time-delays. In IEEE Conf. on Decision and Control, Maui, Hawaii, USA, Dec. 2012.

[17] E. Nuño, I. Sarras, and L. Basañez. Consensus in networks of nonidentical Euler-Lagrange systems using $\mathrm{P}+\mathrm{d}$ controllers. IEEE Transactions on Robotics, 26(6):1503-1508, 2013.

[18] M. Ye, B.D.O. Anderson, and C. Yu. Distributed modelindependent consensus of Euler-Lagrange agents on directed networks. International Journal of Robust and Nonlinear Control, 27(14):2428-2450, sep 2017.

[19] A. Abdessameud, A. Tayebi, and I.G. Polushin. Leader-follower synchronization of Euler- Lagrange systems with time-varying leader trajectory and constrained discrete-time communication. IEEE Transactions on Automatic Control, 62(5):2539-2545, 2017.

[20] A. Abdessameud, I.G. Polushin, and A. Tayebi. Synchronization of Lagrangian systems with irregular communication delays. IEEE Transactions on Automatic Control, 59(1):187-193, 2014.

[21] R. Kelly, V. Santibañez, and A. Loria. Control of robot manipulators in joint space. Springer-Verlag, 2005.

[22] A. Astolfi, D. Karagiannis, and R. Ortega. Nonlinear and adaptive control design and applications. Springer-Verlag, 2007.
[23] R. Ortega, A. Loria, P.J. Nicklasson, and H.J. Sira-Ramirez. Passivity-based Control of Euler-Lagrange Systems: Mechanical, Electrical and Electromechanical Applications. Springer, 1998.

[24] F. Ghorbel, B. Srinivasan, and M.W. Spong. On the uniform boundedness of the inertia matrix of serial robot manipulators. Journal of Robotic Systems, 15(1):17-28, 1998.

[25] Y. Hong, J. Hu, and L. Gao. Tracking control for multi-agent consensus with an active leader and variable topology. Automatica, 42(7):1177-1182, 2006.

[26] K. Gu, V.L. Kharitonov, and J. Chen. Stability of Time-Delay Systems. Birkhauser, Boston, 2003.

[27] N. N. Krasovskiu. Stability of motion. Stanford Univ. Press, 1963. Translation of Russian edition, Moscow 1959.

[28] A. Abdessameud and A. Tayebi. Formation control of VTOL unmanned aerial vehicles with communication delays. Automatica, 47(11):2383-2394, 2011.

[29] A. Abdessameud and A. Tayebi. Synchronization of networked Lagrangian systems with input constraints. In Proc. of the 18th IFAC world congress, Milan, Italy, pp. 2382-2387.

[30] A. Seuret and D. V. Dimarogonas and K. H. Johansson. Consensus of double integrator multi-agents under communication delay. IFAC Proceedings Volumes, 42(14):376-381, 2009. 